\title{
ERRATUM
}

\section{Erratum to: A note on the McShane's identity for Hecke groups}

\section{K. Farooq}

Published online: 5 August 2021

(C) The Indian National Science Academy 2021

Correction to: Indian Journal of Pure and Applied Mathematics (2021) https://doi.org/10.1007/s13226-021-00043-6

Due to a typesetting mistake, the PDF version of this article was published with an error in Theorem A. It must read

$$
\sum_{\mathcal{P}_{\alpha, \zeta(\alpha)}^{h}, \mathcal{P}_{\alpha}^{h}, \mathcal{P}_{\alpha, \zeta(\alpha)}} 2 \arctan \left[\frac{1}{\exp \left(\frac{|h|}{4}+\frac{|\alpha|}{2}\right)}\right]=\frac{\pi}{2} .
$$

This has been corrected. No other changes to the article have been made. We apologise for any inconvenience caused to our readers.

The original article can be found online at https://doi.org/10.1007/s13226-021-00043-6.

K. Farooq $(\bowtie)$

FAST, National University of Computer and Emerging Sciences, Islamabad, Pakistan

E-mail: s.khadija.farooq@gmail.com 